\title{
ON STOCHASTIC SUBDIFFERENTIAL SYSTEMS DRIVEN BY STANDARD BROWNIAN MOTION
}

\author{
YONG HUANG, BIAO ZENG, AND JING ZHAO
}

Received 24 May, 2015

\begin{abstract}
The main purpose of this paper is to study the existence of solutions of stochastic subdifferential inclusions systems driven by standard Brownian motion. We first use the subjectivity theorem to obtain the existence result of subdifferential inclusion, and then, by applying the Banach contraction principle we obtain the solution of the corresponding stochastic system. Our results are inspired by the one in which the existence and uniqueness of solutions to the subdifferential inclusion system is given.
\end{abstract}

2010 Mathematics Subject Classification: 34B10; 34B15

Keywords: subdifferential inclusions, pseudomonotonicity, subjectivity theorem, Brownian motion

\section{INTRODUCTION}

The theory of stochastic system has been rising as an important field of study in recent decades. A great number of applications to many problems in control theory, biology, physics, mechanics and electrical engineering can be modelled by stochastic system; see $[2,5,11,12,17,19,20]$ and the references cited therein. Furthermore, the evolution inclusions play a significant role as a tool in the investigation of different dynamical process and phenomena represented by the inclusion with a discontinuous or multi-valued right-hand side, arising, specifically, in the investigation of dynamics of economical, dynamic Coulomb friction problems and biological macrosystems. Also, they are extremely valuable in demonstrating existence theorems in control theory and differential variational inequalities. Stochastic evolution inclusions in infinite dimensional spaces, have not been considered, particular, stochastic evolution inclusions of Clarke's subdifferential type.

The first author was supported in part by NSF of Guangxi Grant No. 2013GXNSFAA019022, Scientific Research Fund of Guangxi Education Department Grant No. 2013 YB243.

The third author was supported in part by NNSF of China Grant Nos. 11271087, 61263006, NSF of Guangxi Grant Nos. 2014GXNSFDA118002, 2015GXNSFAA309018, 2014GXNSFBA118005 and Special Funds of Guangxi Distinguished Experts Construction Engineering.

(c) 2016 Miskolc University Press 
The study for the existence of evolution systems described by the inclusions of Clarke's subdifferential type is still untreated topic in the literature and this fact is the motivation of the present work. In fact, the Clarke's subdifferential has important applications in mechanics and engineering, especially in nonsmooth analysis and optimization (see [1,15]). The evolution inclusions with Clarke's subdifferential type have been studied in many papers (see [3,4, 6-10,13-16, 18]).

To the best of our knowledge, there is no paper which investigates the study of stochastic evolution inclusions driven by a standard Brownian motion. Thus, we will make the first attempt to study such problems in this paper. Our results are inspired by the one in where the existence and uniqueness of solutions to the system is given (see Chapter 4 of [15]).

The rest of this paper is organized as follows, In Section 2 we introduce some notations, concepts, and basic results about standard Brownian motion and we recall some preliminary results about Clarke's Subdifferential and pseudomonotone operators. The existence and uniqueness of solutions are discussed in Section 3 by using the subjectivity theorem and Banach contraction principle.

\section{PReliminaries}

Let $H$ be a reflexive Banach space. Let $(\Omega, \Gamma, P)$ be a complete probability space with probability measure $P$ on $\Omega$ and a filtration $\left\{\Gamma_{t} \mid t \in[0, T]\right\}$ generated by the $H$ valued Wiener process $\{W(s): 0 \leq s \leq t\}$ defined on the probability space $(\Omega, \Gamma, P)$ and $\Gamma_{T}=\Gamma$. Let $L_{2}\left(\Omega, \Gamma_{T}, H\right)$ be the Hilbert space of all $\Gamma_{T}$-measurable square integrable random variables with values in $H$. Moreover, let $L^{2}\left(0, T ; L_{2}\left(\Omega, \Gamma_{T}, H\right)\right)$ be the Banach space of continuous maps from $(0, T)$ into $L_{2}\left(\Omega, \Gamma_{T}, H\right)$ satisfying $E \int_{0}^{T}\|x(t)\|^{2} d t<+\infty$. Clearly, $L^{2}\left(0, T ; L_{2}\left(\Omega, \Gamma_{T}, H\right)\right)$ is a Banach space with norm $\|x\|_{2}=\left(E \int_{0}^{T}\|x(t)\|^{2} d t\right)^{1 / 2}$.

Let $X$ be a Banach space with the norm denoted by $\|\cdot\|_{X}$. Denote by $X^{*}$ its dual space and $\langle\cdot, \cdot\rangle_{X}{ }^{*} \times X$ the duality pairing between $X^{*}$ and $X$. We specify the strong convergence by $\rightarrow$ and the weak convergence by $\rightarrow$.

Now, we give some basic preliminaries which can be founded in [15].

Definition 1. Let $F: X \rightrightarrows X^{*}$ be a multi-valued mapping. Then $F$ is said to be

(i) monotone, if for each $x, y \in X$,

$$
\left\langle y^{*}-x^{*}, y-x\right\rangle \geq 0, \forall x^{*} \in F(x) \text { and } y^{*} \in F(y) .
$$

(ii) maximal monotone, if the graph of the monotone mapping $F$ is not included in the graph of any other monotone mapping with the same domain.

(iii) pseudomonotone, if

(a) for each $x \in X$, the set $F(x)$ is nonempty, bounded, closed and convex;

(b) the mapping $F$ is u.s.c. from each finite-dimensional subspace of $X$ to $X^{*}$ endowed with the weak topology; 
(c) if $\left\{x_{n}\right\} \subset X$ with $x_{n} \rightarrow x$ in $X$, and $x_{n}^{*} \in F\left(x_{n}\right)$ is such that

$$
\limsup _{n \rightarrow \infty}\left\langle x_{n}^{*}, x_{n}-x\right\rangle \leq 0,
$$

then for every $y \in X$, there exists $x^{*}(y) \in F(x)$ such that

$$
\left\langle x^{*}(y), x-y\right\rangle \leq \liminf _{n \rightarrow \infty}\left\langle x_{n}^{*}, x_{n}-y\right\rangle .
$$

(iv) generalized pseudomonotone, if for any sequence $\left\{x_{n}\right\} \subset X$ with $x_{n} \rightarrow x$ in $X$, and $x_{n}^{*} \in F\left(x_{n}\right)$ with $x_{n}^{*} \rightarrow x^{*}$ such that

$$
\limsup _{n \rightarrow \infty}\left\langle x_{n}^{*}, x_{n}-x\right\rangle \leq 0,
$$

we have $x^{*} \in F(x)$ and $\left\langle x_{n}^{*}, x_{n}\right\rangle \rightarrow\left\langle x^{*}, x\right\rangle$.

Definition 2. Let $F: X \rightarrow X^{*}$ be a single-valued mapping. Then $F$ is said to be pseudomonotone, if it is bounded and satisfies the inequality

$$
\langle F x, x-y\rangle \leq \liminf _{n \rightarrow \infty}\left\langle F x_{n}, x_{n}-y\right\rangle \quad \text { for all } x \in X,
$$

where $x_{n} \rightarrow x$ in $X$ with

$$
\limsup _{n \rightarrow \infty}\left\langle F x_{n}, x_{n}-x\right\rangle \leq 0 .
$$

Definition 3. Let $F: X \rightrightarrows X^{*}$ be a multi-valued mapping. We say that $F$ is coercive if either $D(F)$ (the domain of $F$ ) is bounded or $D(F)$ is unbounded and

$$
\lim _{\|x\|_{X} \rightarrow \infty, x \in D(F)} \frac{\inf \left\{\left\langle x^{*}, x\right\rangle_{X^{*} \times X} \mid x^{*} \in F(x)\right\}}{\|x\|_{X}}=+\infty .
$$

Definition 4. Let $F: X \rightarrow X^{*}$ be a single-valued mapping. We say that $F$ is coercive if

$$
\lim _{\|x\|_{X} \rightarrow \infty} \frac{\langle F x, x\rangle_{X^{*} \times X}}{\|x\|_{X}}=+\infty .
$$

Moreover, $F$ is said to be coercive with constant $\alpha$ if there exists $\alpha>0$ such that

$$
\langle F x, x\rangle_{X^{*} \times X} \geq \alpha\|x\|_{X}^{2} \quad \text { for all } x \in X .
$$

Theorem 1 (Subjectivity Theorem, [15]). Let $X$ be a reflexive Banach space and $F: X \rightrightarrows X^{*}$ be pseudomonotone and coercive. Then $F$ is surjective, i.e., $R(F)=$ $X^{*}$.

Next, we proceed with the definition of the Clarke subdifferential for a locally Lipschitz function $j: X \rightarrow \mathbb{R}$ (one can see $[1,15]$ ). We denote by $j^{0}(x ; v)$ the Clarke generalized directional derivative of $j$ at the point $x \in X$ in the direction $v \in X$, that is

$$
j^{0}(x ; v):=\limsup _{\lambda \rightarrow 0^{+}, \zeta \rightarrow x} \frac{j(\zeta+\lambda v)-j(\zeta)}{\lambda} .
$$


Recall also that the Clarke subdifferential or generalized gradient of $j$ at $x \in X$, denoted by $\partial j(x)$, is a subset of $X^{*}$ given by

$$
\partial j(x):=\left\{x^{*} \in X^{*} \mid j^{0}(x ; v) \geq\left\langle x^{*}, v\right\rangle \text { for all } v \in X\right\} .
$$

Lemma 1 ([15], Proposition 3.23). If $j: X \rightarrow \mathbb{R}$ is locally Lipschitz function on a subset $E$ of $X$, then

(i) the function $(x, v) \mapsto j^{0}(x ; v)$ is u.s.c. from $E \times X$ into $\mathbb{R}$;

(ii) for every $x \in E$ the subgradient $\partial j(x)$ is a nonempty, convex, and weakly* compact subset of $X^{*}$ which is bounded by the Lipschitz constant $K_{x}>0$ of $j$ near $x$;

(iii) the graph of $\partial j$ is closed in $X \times X_{w^{*}}^{*}$;

(iv) the multi-valued mapping $\partial j$ is u.s.c. from $E$ into $X_{w^{*}}^{*}$, i.e., $\partial j$ is weakly u.s.c. from $E$ into $X^{*}$.

\section{EXISTENCE RESULTS}

Let $X$ be a reflexive Banach space, $Y$ be a Banach space and $M: X \rightarrow Y$ be a given linear continuous operator. We denote by $M^{*}: Y^{*} \rightarrow X^{*}$ the adjoint operator to $M$. Let $A:(0, T) \times X \rightarrow X^{*}, J:(0, T) \times X \rightarrow \mathbb{R}$ and $f:(0, T) \rightarrow X^{*}$ be given. Let $X=L^{2}(0, T ; X)$. It is clear that $X^{*}=L^{2}\left(0, T ; X^{*}\right)$.

We first consider the subdifferential inclusion problem of the following problem: Find $x \in \mathcal{X}$ such that

$$
A(t, x(t))+M^{*} \partial J(t, M x(t)) \ni f(t) \text { a.e. } t \in(0, T),
$$

where $f \in X^{*}$ and $\partial J(t, \cdot)$ denotes the Clarke subdifferential of $J(t, \cdot)$

Definition 5. A function $x \in \mathcal{X}$ is called a solution to problem (3.1) if and only if there exists $\zeta \in \mathcal{X}^{*}$ such that

$$
\left\{\begin{array}{l}
A(t, x(t))+\zeta(t)=f(t) \text { a.e. } t \in(0, T), \\
\zeta(t) \in M^{*} \partial J(t, M x(t)) \text { a.e. } t \in(0, T) .
\end{array}\right.
$$

We will make the following hypotheses:

$\left(H_{A}\right) A:(0, T) \times X \rightarrow X^{*}$ is such that

(i) $A(\cdot, x)$ is measurable on $(0, T)$ for all $x \in X$;

(ii) $A(t, \cdot)$ is pseudomonotone and coercive with constant $\alpha>0$, for a.e. $t \in$ $(0, T)$;

(iii) $A(t, \cdot)$ is strongly monotone for a.e. $t \in(0, T)$, i.e.,

$$
\left\langle A\left(t, x_{1}\right)-A\left(t, x_{2}\right), x_{1}-x_{2}\right\rangle \geq m_{1}\left\|x_{1}-x_{2}\right\|^{2}
$$

for all $x_{1}, x_{2} \in X$, a.e. $t \in(0, T)$ with $m_{1}>0$.

$\left(H_{J}\right) J:(0, T) \times X \rightarrow \mathbb{R}$ is such that

(i) $J(\cdot, x)$ is measurable on $(0, T)$ for all $x \in X$; 
(ii) $J(t, \cdot)$ is locally Lipschitz on $X$ for a.e. $t \in(0, T)$;

(iii) $\|\partial J(t, x)\|_{X^{*}} \leq c_{0}+c_{1}\|x\|_{X}$ for all $x \in X$, a.e. $t \in(0, T)$ with $c_{0}, c_{1}>0$;

(iv) $\left\langle z_{1}-z_{2}, x_{1}-x_{2}\right\rangle \geq-m_{2}\left\|x_{1}-x_{2}\right\|^{2}$ for all $z_{i} \in \partial J\left(t, x_{i}\right), x_{i} \in X, i=1,2$, a.e. $t \in(0, T)$ with $m_{2}>0$;

(v) $J^{\circ}(t, x ;-x) \leq d_{0}(1+\|x\|)$ for all $x \in X$, a.e. $t \in(0, T)$ with $d_{0}>0$.

$\left(H_{M}\right) M: X \rightarrow Y$ is compact.

From the idea of Theorem 4.11 of [15], we have the following result.

Theorem 2. Assume that hypotheses $\left(H_{A}\right),\left(H_{M}\right)$ hold. If one of the following hypotheses:

(a) $\left(H_{J}\right)(i)$-(iv) and $\alpha>c_{1}\|M\|^{2}$,

(b) $\left(H_{J}\right)$,

is satisfied and $m_{1}>m_{2}\|M\|^{2}$ holds, then problem (3.1) has a unique solution $x \in$ $X$. Moreover, the solution satisfies

$$
\|x\| x \leq c\left(1+\|f\|_{X^{*}}\right)
$$

with some constant $c>0$.

Proof. Let $t \in(0, T)$ be fixed. We define the multi-valued mapping $F_{t}: X \rightrightarrows X^{*}$ by

$$
F_{t}(x)=A(t, x)+M^{*} \partial J(t, M x)
$$

for $x \in X$. We will show that $F_{t}$ is pseudomonotone and coercive. First, we prove the pseudomonotonicity of $F_{t}$ and, to this end, we use Proposition 3.58 of [15] which states that a generalized pseudomonotone operator which is bounded and has nonempty, closed and convex values is pseudomonotone.

From Lemma 1 , it is clear that $F_{t}$ has nonempty, closed and convex values in $X^{*}$. From the boundedness of $A(t, \cdot)$ and (iii) of $\left(H_{J}\right)$, it follows that $F_{t}$ is a bounded map.

We now show that $F_{t}$ is a generalized pseudomonotone operator. Let $x_{n}, x \in$ $X, x_{n} \rightarrow x$ in $X, x_{n}^{*}, x^{*} \in X^{*}, x_{n}^{*} \rightarrow x^{*}$ in $X^{*}, x_{n}^{*} \in F_{t}\left(x_{n}\right)$ and assume that

$$
\lim \sup \left\langle x_{n}^{*}, x_{n}-x\right\rangle \leq 0 .
$$

We prove that $x^{*} \in F_{t}(x)$ and

$$
\left\langle x_{n}^{*}, x_{n}\right\rangle \rightarrow\left\langle x^{*}, x\right\rangle \text {. }
$$

We have $x_{n}^{*}=A\left(t, x_{n}\right)+\zeta_{n}$ with $\zeta_{n}=M^{*} z_{n}, z_{n} \in \partial J\left(t, M x_{n}\right)$. From the compactness of $M$ it follows that $M x_{n} \rightarrow M x$ in $Y$. From (iii) of $\left(H_{J}\right)$, passing to a subsequence if necessary, we have $z_{n} \rightarrow z$ in $Y^{*}$ with some $z \in Y^{*}$, hence $\zeta_{n} \rightarrow \zeta$ with some $\zeta \in X^{*}$. By (iii) of Lemma 1 , since $z_{n} \in \partial J\left(t, M x_{n}\right)$, we deduce that $\zeta=M^{*} z \in M^{*} \partial J(t, M x)$. Furthermore, from the equality

$$
\begin{aligned}
\left\langle x_{n}^{*}, x_{n}-x\right\rangle & =\left\langle A\left(t, x_{n}\right), x_{n}-x\right\rangle+\left\langle\zeta_{n}, x_{n}-x\right\rangle \\
& =\left\langle A\left(t, x_{n}\right), x_{n}-x\right\rangle+\left\langle z_{n}, M x_{n}-M x\right\rangle_{Y^{*} \times Y},
\end{aligned}
$$


we obtain

$$
\limsup \left\langle A\left(t, x_{n}\right), x_{n}-x\right\rangle=\limsup \left\langle x_{n}^{*}, x_{n}-x\right\rangle \leq 0 .
$$

Applying the pseudomonotonicity of $A(t, \cdot)$, by Proposition 3.66 of [15] we deduce that $A\left(t, x_{n}\right) \rightarrow A(t, x)$ in $X^{*}$ and $\lim \left\langle A\left(t, x_{n}\right), x_{n}-x\right\rangle=0$. Therefore, passing to the limit in the equation $x_{n}^{*}=A\left(t, x_{n}\right)+\zeta_{n}$, we have $x^{*}=A(t, x)+\zeta$ which, together with $\zeta \in M^{*} \partial J(t, M x)$, implies $x^{*} \in A(t, x)+M^{*} \partial J(t, M x)=F_{t} x$. Next, from above we obtain

$$
\begin{aligned}
\lim \left\langle x_{n}^{*}, x_{n}\right\rangle & =\lim \left\langle A\left(t, x_{n}\right), x_{n}-x\right\rangle+\lim \left\langle A\left(t, x_{n}\right), x\right\rangle+\lim \left\langle\zeta_{n}, x_{n}\right\rangle \\
& =\langle A(t, x), x\rangle+\langle\zeta, x\rangle=\left\langle x^{*}, x\right\rangle
\end{aligned}
$$

which, according to Definition 1 , shows that $F$ is a generalized pseudomonotone operator.

Next, from (iii) of $\left(H_{J}\right)$ we have

$$
\begin{aligned}
\left\|M^{*} \partial J(t, M x)\right\| & \leq\left\|M^{*}\right\|\|\partial J(t, M x)\|_{Y^{*}} \\
& \leq\left\|M^{*}\right\|\left(c_{0}+c_{1}\|M\|\|x\|_{X}\right), \\
\left|\left\langle M^{*} \partial J(t, M x), x\right\rangle\right| & \leq\left\|M^{*} \partial J(t, M x)\right\|\|x\| \\
& \leq c_{1}\|M\|^{2}\|x\|^{2}+c_{0}\|M\|\|x\|,
\end{aligned}
$$

hence

$$
\left\langle M^{*} \partial J(t, M x), x\right\rangle \geq-c_{1}\|M\|^{2}\|x\|^{2}-c_{0}\|M\|\|x\| .
$$

Therefore, since $A(t, \cdot)$ is coercive with constant $\alpha>0$, we have

$$
\begin{aligned}
\left\langle F_{x} x, x\right\rangle & =\langle A(t, x), x\rangle+\left\langle M^{*} \partial J(t, M x), x\right\rangle \\
& \geq\left(\alpha-c_{1}\|M\|^{2}\right)\|x\|^{2}-c_{0}\|M\|\|x\|
\end{aligned}
$$

for all $x \in X$.

In case (a), $\alpha>c_{1}\|M\|^{2}$, hence

$$
\lim _{\|x\| \rightarrow \infty} \frac{\inf \left\{\left\langle x^{*}, x\right\rangle \mid x^{*} \in F_{t} x\right\}}{\|x\|}=+\infty .
$$

In case (b), let $x \in X$ and $\zeta \in M^{*} \partial J(t, M x)$. So $\zeta=M^{*} z$ and $z \in \partial J(t, M x)$. From (iv) of $\left(H_{J}\right)$ we have

$$
-\langle z, M x\rangle_{Y^{*} \times Y} \leq J^{\circ}(t, M x ;-M x) \leq d_{0}(1+\|M x\|) \leq d_{0}(1+\|M\|\|x\|) .
$$

Hence, it follows that

$$
\langle\zeta, x\rangle_{Y^{*} \times Y}=\left\langle M^{*} z, x\right\rangle=\langle z, M x\rangle_{Y^{*} \times Y} \geq-d_{0}(1+\|M\|\|x\|),
$$

which shows that (3.3) is also true. Then the operator $F_{t}$ is coercive.

Applying Theorem 1 we deduce that $F_{t}$ is surjective, which implies that the following problem

$$
A(t, x)+M^{*} \partial J(t, M x) \ni f \text { a.e. } t \in(0, T)
$$


has a solution $x \in X$, where $f \in X^{*}$. Moreover, from the coercivity of $F_{t}$, we have

$$
\left(\alpha-c_{1}\|M\|^{2}\right)\|x\|_{X}^{2}-c_{0}\|M\|\|x\|_{X} \leq\|f\|_{X^{*}}\|x\|_{X}
$$

which implies that the following estimate

$$
\|x\|_{X} \leq c\left(1+\|f\|_{X^{*}}\right)
$$

holds with a positive constant $c$ depending on $c_{0}, c_{1}$ and $\alpha$.

We now prove the uniqueness to problem (3.4). Let $x_{1}, x_{2} \in X$ be s solution to problem (3.4). Then there exist $z_{i} \in Y^{*}$ and $z_{i} \in \partial J\left(t, M x_{i}\right)$ such that

$$
A\left(t, x_{i}\right)+M^{*} z_{i}=f \text { for } i=1,2 .
$$

Subtracting the above two equations, multiplying the result by $x_{1}-x_{2}$, and using the strong monotonicity of $A(t, \cdot)$, we have

$$
m_{1}\left\|x_{1}-x_{2}\right\|^{2}+\left\langle M^{*} z_{1}-M^{*} z_{2}, x_{1}-x_{2}\right\rangle \leq 0 .
$$

Next, by (iv) of $\left(H_{J}\right)$ and $m_{1}>m_{2}\|M\|^{2}$, we obtain

$$
\begin{aligned}
\left\langle M^{*} z_{1}-M^{*} z_{2}, x_{1}-x_{2}\right\rangle & =\left\langle z_{1}-z_{2}, M x_{1}-M x_{2}\right\rangle_{Y^{*} \times Y} \\
& \geq-m_{2}\left\|M x_{1}-M x_{2}\right\|_{Y}^{2} \\
& \geq-m_{2}\|M\|^{2}\left\|x_{1}-x_{2}\right\|_{X}^{2} .
\end{aligned}
$$

Combine (3.6) and (3.7) we obtain

$$
\left(m_{1}-m_{2}\|M\|^{2}\right)\left\|x_{1}-x_{2}\right\|_{X}^{2} \leq 0,
$$

which in view of $m_{1}>m_{2}\|M\|^{2}$, implies $x_{1}=x_{2}$. Subsequently, from (3.5) we deduce that $z_{1}=z_{2}$ which completes the proof of the uniqueness. Therefore, we deduce that for a.e. $t \in(0, T)$ problem (3.1) has a unique solution $x(t) \in X$ and, moreover,

$$
\|x(t)\|_{X} \leq c\left(1+\|f(t)\|_{X^{*}}\right)
$$

for a.e. $t \in(0, T)$ with $c>0$. We point out the constant $c$ is independent of $t$.

We prove that the function $t \mapsto x(t)$ defined above is measurable on $(0, T)$. To this end, given $g \in X^{*}$ we denote by $\omega \in X$ the unique solution of the inclusion

$$
A(t, \omega)+M^{*} \partial J(t, M \omega) \ni g \text { a.e. } t \in(0, T) .
$$

Since $A$ and $J$ depend on the parameter $t$, the solution $\omega$ is also a function of $t$, i.e., $\omega=\omega(t)$. We claim that the solution $\omega$ depends continuously on the right-side $g$, for a.e. $t \in(0, T)$. Indeed, let $g_{1}, g_{2} \in X^{*}$ and $\omega_{1}(t), \omega_{2}(t) \in X$ be the corresponding solutions to problem (3.9). From Definition 5 we have

$$
\begin{gathered}
A\left(t, \omega_{1}(t)\right)+\zeta_{1}(t)=g_{1} \text { a.e. } t \in(0, T), \\
A\left(t, \omega_{2}(t)\right)+\zeta_{2}(t)=g_{2} \text { a.e. } t \in(0, T), \\
\zeta_{1}(t) \in M^{*} \partial J\left(t, M \omega_{1}(t)\right), \zeta_{2}(t) \in M^{*} \partial J\left(t, M \omega_{2}(t)\right) \text { a.e. } t \in(0, T) .
\end{gathered}
$$

Subtracting (3.11) from (3.10), multiplying the result by $\omega_{1}(t)-\omega_{2}(t)$, we get 


$$
\begin{aligned}
&\left\langle A\left(t, \omega_{1}(t)\right)-A\left(t, \omega_{2}(t)\right), \omega_{1}(t)-\omega_{2}(t)\right\rangle+\left\langle\zeta_{1}(t)-\zeta_{2}(t), \omega_{1}(t)-\omega_{2}(t)\right\rangle \\
&=\left\langle g_{1}-g_{2}, \omega_{1}(t)-\omega_{2}(t)\right\rangle
\end{aligned}
$$

for a.e. $t \in(0, T)$. Since $\zeta_{i}(t) \in M^{*} z_{i}(t)$ with $z_{i}(t) \in \partial J\left(t, M \omega_{i}(t)\right)$ for a.e. $t \in$ $(0, T)$ and $i=1,2$, by (iii) of $\left(H_{A}\right)$ and (iv) of $\left(H_{J}\right)$, we obtain

$m_{1}\left\|\omega_{1}(t)-\omega_{2}(t)\right\|_{X}^{2}-m_{2}\|M\|^{2}\left\|\omega_{1}(t)-\omega_{2}(t)\right\|_{X}^{2}=\left\|g_{1}-g_{2}\right\|_{X^{*}}\left\|\omega_{1}(t)-\omega_{2}(t)\right\|_{X}$ for a.e. $t \in(0, T)$. Exploiting $m_{1}>m_{2}\|M\|^{2}$, we get

$$
\left\|\omega_{1}(t)-\omega_{2}(t)\right\|^{2} \leq \widetilde{c}\left\|g_{1}-g_{2}\right\|_{X^{*}} \text { for a.e. } t \in(0, T),
$$

where $\widetilde{c}=\left(m_{1}-m_{2}\|M\|^{2}\right)^{-1}$ is independent of $t$. Hence, we have that the mapping $X^{*} \ni g \mapsto \omega(t) \in X$ is continuous, for a.e. $t \in(0, T)$, which proves the claim. Now, by (3.12) and the measurability of $f$, we deduce that the solution $x$ of problem (3.1) is measurable on $(0, T)$. Since $f \in X^{*}$, from the estimate (3.8), we conclude that $x \in \mathcal{X}$, and moreover, (3.2) holds. The proof of the theorem is complete.

Let $X=L_{2}\left(\Omega, \Gamma_{T}, H\right), \mathcal{X}=L^{2}\left(0, T ; L_{2}\left(\Omega, \Gamma_{T}, H\right)\right)$. We now study the stochastic subdifferential inclusion problem having the following form:

Find $x \in \mathcal{X}$ such that

$$
A(t, x(t))+\int_{0}^{t} \sigma(s, x(s)) d W(s)+M^{*} \partial J(t, M x(t)) \ni f(t) \text { a.e. } t \in(0, T),
$$

where $W$ is a standard Brownian motion and $\sigma:(0, T) \times X \rightarrow L^{2}\left(H ; X^{*}\right)$.

Definition 6. A function $x \in X$ is called a solution to problem (3.13) if and only if there exists $\zeta \in \mathcal{X}^{*}$ such that

$$
\left\{\begin{array}{l}
A(t, x(t))+\int_{0}^{t} \sigma(s, x(s)) d W(s)+\zeta(t)=f(t) \text { a.e. } t \in(0, T), \\
\zeta(t) \in M^{*} \partial J(t, M x(t)) \text { a.e. } t \in(0, T)
\end{array}\right.
$$

We need the following hypothesis.

$\left(H_{\sigma}\right)$ There exist constants $l_{1}, l_{2}>0$ such that

$$
\|\sigma(t, x)\|^{2} \leq l_{1}(1+\|x\|),\left\|\sigma\left(t, x_{1}\right)-\sigma\left(t, x_{2}\right)\right\|^{2} \leq l_{2}\left\|x_{1}-x_{2}\right\|
$$

for all $x, x_{1}, x_{2} \in X$.

Theorem 3. Assume that hypotheses $\left(H_{A}\right),\left(H_{M}\right),\left(H_{\sigma}\right)$ hold. If one of the following hypotheses:

(a) $\left(H_{J}\right)(i)$-(iv) and $\alpha>c_{1}\|M\|^{2}$,

(b) $\left(H_{J}\right)$,

is satisfied and $m_{1}>m_{2}\|M\|^{2}$ holds, then problem (3.13) has a unique solution in $x$. 
Proof. Let $\eta \in \mathcal{X}^{*}$, We denote by $x_{\eta} \in \mathcal{X}$ the solution of the inclusion

$$
A\left(t, x_{\eta}(t)\right)+M^{*} \partial J\left(t, M x_{\eta}(t)\right) \ni f(t)-\eta(t) \text { a.e. } t \in(0, T),
$$

guaranteed by Theorem 2 . We know that $x_{\eta} \in \mathcal{X}$ is unique and it satisfies

$$
\left\|x_{\eta}\right\| x \leq c\left(1+\|f\|_{X^{*}}+\|\eta\|_{X^{*}}\right)
$$

with $c>0$. We consider the operator $\Lambda: X^{*} \rightarrow X^{*}$ defined by

$$
(\Lambda \eta)(t)=\int_{0}^{t} \sigma\left(s, x_{\eta}(s)\right) d W(s) \text { for all } \eta \in \mathcal{X}^{*} \text {, a.e. } t \in(0, T) .
$$

It is easy to check that the operator $\Lambda$ is well defined. Indeed, for $\eta \in X^{*}$, From $\left(H_{\sigma}\right)$ we have

$$
\begin{aligned}
E\left\|\int_{0}^{t} \sigma\left(s, x_{\eta}(s)\right) d W(s)\right\|^{2} & =\int_{0}^{t} E\left\|\sigma\left(s, x_{\eta}(s)\right)\right\|^{2} d s \\
& \leq \int_{0}^{t} l_{1}\left(1+E\left\|x_{\eta}(s)\right\|^{2}\right) d s
\end{aligned}
$$

for a.e. $t \in(0, T)$. From (3.15) we obtain that the integral in (3.16) is well defined and the operator $\Lambda$ takes valued in $X^{*}$.

Next, we show that $\Lambda$ has a unique fixed point. Let $\eta_{1}, \eta_{2} \in X^{*}$ and let $x_{1}=x_{\eta_{1}}$ and $x_{2}=x_{\eta_{2}}$ be the corresponding solutions to (3.14). We have $x_{1}, x_{2} \in \mathcal{X}$ and

$$
\begin{gathered}
A\left(t, x_{1}(t)\right)+\zeta_{1}(t)=f(t)-\eta_{1}(t) \text { a.e. } t \in(0, T), \\
A\left(t, x_{2}(t)\right)+\zeta_{2}(t)=f(t)-\eta_{2}(t) \text { a.e. } t \in(0, T), \\
\zeta_{1}(t) \in M^{*} \partial J\left(t, M x_{1}(t)\right), \zeta_{2}(t) \in M^{*} \partial J\left(t, M x_{2}(t)\right) \text { a.e. } t \in(0, T) .
\end{gathered}
$$

Subtracting (3.18) from (3.17), multiplying the result by $x_{1}(t)-x_{2}(t)$ and using (iii) of $\left(H_{A}\right)$, (iv) of $\left(H_{J}\right)$, we obtain

$$
\left\|x_{1}(t)-x_{2}(t)\right\|_{X} \leq \widetilde{c}\left\|\eta_{1}(t)-\eta_{2}(t)\right\|_{X^{*}} \text { for a.e. } t \in(0, T)
$$

with $\widetilde{c}>0$. From (3.19), for $t \in(0, T)$ we have

$$
\begin{aligned}
E\left\|\left(\Lambda \eta_{1}\right)(t)-\left(\Lambda \eta_{2}\right)(t)\right\|^{2} & =E\left\|\int_{0}^{t}\left(\sigma\left(s, x_{1}(s)\right)-\sigma\left(s, x_{2}(s)\right)\right) d W(s)\right\|^{2} \\
& =\int_{0}^{t} E\left\|\sigma\left(s, x_{1}(s)\right)-\sigma\left(s, x_{2}(s)\right)\right\|^{2} d s \\
& \leq l_{2} \int_{0}^{t} E\left\|x_{1}(s)-x_{2}(s)\right\|^{2} d s \\
& \leq l_{2} \widetilde{c}^{2} \int_{0}^{t} E\left\|\eta_{1}(s)-\eta_{2}(s)\right\|^{2} d s,
\end{aligned}
$$

and consequently,

$$
\left.E\left\|\left(\Lambda^{2} \eta_{1}\right)(t)-\left(\Lambda^{2} \eta_{2}\right)(t)\right\|^{2} \leq l_{2} \widetilde{c}^{2} \int_{0}^{t} E \| \Lambda \eta_{1}\right)(s)-\left(\Lambda \eta_{2}\right)(s) \|^{2} d s
$$




$$
\begin{aligned}
& \leq l_{2}^{2 \widetilde{c}^{4}} \int_{0}^{t} \int_{0}^{s} E\left\|\eta_{1}(\tau)-\eta_{2}(\tau)\right\|^{2} d \tau d s \\
& \leq l_{2}^{2} \widetilde{c}^{4} \int_{0}^{t} E\left\|\eta_{1}(s)-\eta_{2}(s)\right\|^{2} d s \int_{0}^{t} d s \\
& =l_{2}^{2} \widetilde{c}^{4} t \int_{0}^{t} E\left\|\eta_{1}(s)-\eta_{2}(s)\right\|^{2} d s .
\end{aligned}
$$

Reiterating this inequality $k$ times leads to

$$
E\left\|\left(\Lambda^{k} \eta_{1}\right)(t)-\left(\Lambda^{k} \eta_{2}\right)(t)\right\|^{2} \leq l_{2}^{k} \widetilde{c}^{2 k} \frac{t^{k-1}}{(k-1) !} \int_{0}^{t} E\left\|\eta_{1}(s)-\eta_{2}(s)\right\|^{2} d s
$$

for a.e. $t \in(0, T)$. This implies that

$$
\left\|\Lambda^{k} \eta_{1}-\Lambda^{k} \eta_{2}\right\|_{X^{*}}^{2} \leq l_{2}^{k} \widetilde{c}^{2 k} \frac{T^{k-1}}{(k-1) !}\left\|\eta_{1}-\eta_{2}\right\|_{X^{*}}^{2} .
$$

It is easy to verify that for $k$ sufficient large $\Lambda^{k}$ is a contraction on $\mathcal{X}^{*}$. Therefore, the Banach contraction principle implies that $\Lambda$ has a unique fixed point $\eta^{*} \in \mathcal{X}^{*}$. Then $x_{\eta^{*}}$ is a solution to problem (3.13), which concludes the existence part of the theorem.

The uniqueness part follows from the uniqueness of the fixed point of $\Lambda$. In fact, let $x \in \mathcal{X}$ be a solution to problem (3.13) and define the element $\eta \in X^{*}$ by

$$
\eta(t)=\int_{0}^{t} \sigma(s, x(s)) d W(s) \text { for all } t \in(0, T) .
$$

It follows that $x$ is the solution to the problem (3.14) and by the uniqueness of solutions to problem (3.14), we obtain $x=x_{\eta}$. This implies $\Lambda \eta=\eta$ and by the uniqueness of the fixed point of $\Lambda$ we have $\eta=\eta^{*}$, hence $x=x_{\eta^{*}}$, which concludes the proof.

\section{REFERENCES}

[1] F. Clarke, Optimization and Nonsmooth Analysis. New York: Wiley, 1983.

[2] T. Duncan, Y. Hu, and B. Pasik-Duncan, "Stochastic Calculus for Fractional Brownian Motion. I. Theory." SIAM Journal Control Optimal, vol. 38, pp. 582-612, 2000, doi: 10.1137/S036301299834171X.

[3] L. Gasinski, Z. Liu, S. Migórski, A. Ochal, and Z. Peng, "Hemivariational inequality approach to evolutionary constrained problems on star-shaped sets," J. Optim. Theory Appl., vol. 164, pp. 514-533, 2015, doi: 10.1007/s10957-014-0587-6.

[4] W. Han, S. Migórski, and M. Sofonea, "A class of variational-hemivariational inequalities with applications to frictional contact problems," SIAM J. Math. Anal., vol. 46, no. 6, pp. 3891-3912, 2014, doi: 10.1137/140963248.

[5] D. Keck and M. Mckibben, "Abstract semilinear stochastic Ito-Volterra integrodifferential equations," Miskolc Mathematical Notes, vol. 2006, pp. 1-22, 2006.

[6] Z. Liu, "Browder-Tikhonov regularization of non-coercive evolution hemivariational inequalities," Inverse Problems, vol. 21, pp. 13-20, 2005, doi: 10.1088/0266-5611/21/1/002. 
[7] Z. Liu, "Existence results for quasilinear parabolic hemivariational inequalities," J. Diff. Equa., vol. 244, pp. 1395-1409, 2008, doi: 10.1016/j.jde.2007.09.001.

[8] Z. Liu and X. Li, "Approximate controllability for a class of hemivariational inequalities," Nonlinear Anal. Real World Appl., vol. 22, pp. 581-591, 2015.

[9] Z. Liu and X. Li, "Approximate controllability of fractional evolution systems with RiemannLiouville fractional derivatives," SIAM Journal on Control and Optimization, vol. 53, no. 4, pp. 1920-1933, 2015, doi: 10.1137/120903853.

[10] Z. Liu and B. Zeng, "Existence and controllability for fractional evolution inclusions of Clarkes subdifferential type," Appl. Math. Comput., vol. 257, pp. 178-189, 2015, doi: 10.1016/j.amc.2014.12.057.

[11] L. Lu and Z. Liu, "Existence and controllability results for stochastic fractional evolution hemivariational inequalities," Appl. Math. Comput., vol. 268, pp. 1164-1176, 2015, doi: 10.1016/j.amc.2015.07.023.

[12] X. Mao, Stochastic Differential Equations and Applications. Chichester: Horwood, 1997.

[13] S. Marano, G. Molica Bisci, and D. Motreanu, "Multiple solutions for a class of elliptic hemivariational inequalities," J. Math. Anal. Appl., vol. 337, no. 1, pp. 85-97, 2008, doi: 10.1016/j.jmaa.2007.03.077.

[14] S. Migórski, A. Ochal, M. Shillor, and M. Sofonea, "A model of a spring-mass-damper system with temperature-dependent friction," European J. Appl. Math., vol. 25, no. 1, pp. 45-64, 2014, doi: 10.1017/S0956792513000272.

[15] S. Migórski, A. Ochal, and M. Sofonea, Nonlinear Inclusions and Hemivariational Inequalities, Models and Analysis of Contact Problems. New York: Springer, 2013, vol. 26.

[16] S. Migórski, A. Ochal, and M. Sofonea, "History-dependent variational-hemivariational inequalities in contact mechanics," Nonlinear Anal. Real World Appl., vol. 22, pp. 604-618, 2015, doi: 10.1016/j.nonrwa.2014.09.021.

[17] B. Oksendal, Stochastic Differential Equations. Berlin: Springer, 2002. doi: 10.1007/978-3642-14394-6'5.

[18] Z. Peng, Z. Liu, and X. Liu, "Boundary hemivariational inequality problems with doubly nonlinear operators," Mathematische Annalen, vol. 356, no. 4, pp. 1339-1358, 2013, doi: 10.1007/s00208012-0884-z.

[19] R. Sakthivel and J. Luo, "Asymptotic stability of impulsive stochastic partial differential equations with infinite delays," J. Math. Anal. Appl., vol. 35, pp. 1-6, 2009, doi: 10.1016/j.jmaa.2009.02.002.

[20] R. Sakthivel and J. Luo, "Asymptotic stability of nonlinear impulsive stochastic differential equations,” Stat. Prob. Lett., vol. 79, pp. 1219-1223, 2009, doi: 10.1016/j.spl.2009.01.011.

\section{Authors' addresses}

\section{Yong Huang}

Baise University, Department of Mathematics, Baise 533000, Guangxi Province, P. R. China

E-mail address: yonghuang99@126.com

Biao Zeng

Jagiellonian University, Faculty of Mathematics and Computer Science, Institute of Computer Science ul. Łojasiewicza 6, 30-348 Krakow, Poland

E-mail address: zengbiao316711602@163.com 
Jing Zhao

Guangxi University for Nationalities, Guangxi Key Laboratory of Universities Optimization Control and Engineering Calculation, and College of Sciences, Nanning 530006, Guangxi Province, P. R. China E-mail address: jingzhao100@126.com 